\title{
Können einzelsprachliche Interventionen sprachenübergreifende Effekte haben? Wie die schulische Majoritätssprache Herkunftssprachen fördern kann
}

\author{
Nicole Marx (D) Torsten Steinhoff
}

Eingegangen: 23. September 2020 / Überarbeitet: 31. Januar 2021 / Angenommen: 15. April 2021 /

Online publiziert: 23. Juli 2021

(C) Der/die Autor(en) 2021

Zusammenfassung Vom herkunftssprachlichen Unterricht wird häufig nicht nur erwartet, dass er herkunfts- und plurilinguale Kompetenzen fördert, sondern auch, dass er der Förderung von Deutschkompetenzen dient. Für den Großteil der Schüler*innen, die zu Hause kein oder nicht nur Deutsch sprechen, erscheint diese Annahme problematisch. Denn sie sind in Deutschland eingeschult worden und erwerben bildungssprachliche Kompetenzen primär auf Deutsch. Diese Einsicht wurde zum Anlass für eine longitudinale Studie in 6. Klassen zu der Frage genommen, wie sich im Schreibunterricht Interventionen in deutscher Sprache auf das Schreiben in türkischer Sprache auswirken und welche sprachenübergreifenden Zusammenhänge dabei zwischen der Textqualität und den Textrevisionen bestehen. Die Ergebnisse deuten darauf hin, dass Teilkompetenzen des Schreibens nicht an Einzelsprachen gebunden, sondern transversal, d.h. in mehreren Sprachen nutzbar sind, und dass Schreibkompetenzen von der schulischen Majoritätssprache in die Herkunftssprache transferiert werden können. Daraus schlussfolgern wir, dass ein sprachenübergreifender Schreibunterricht transversale Teilkompetenzen fokussieren sollte.

Schlüsselwörter Herkunftssprachen · Sprachenübergreifendes Schreiben · Textüberarbeitung $\cdot$ Bilingual $\cdot$ Sprachenübergreifender Transfer

\footnotetext{
Prof. Dr. Nicole Marx $(\bowtie)$

Institut für Deutsche Sprache und Literatur II, Mercator Institut für Sprachförderung und Deutsch als Zweitsprache, Universität zu Köln, Albertus-Magnus-Platz, 50923 Köln, Deutschland

E-Mail: n.marx@uni-koeln.de

Prof. Dr. Torsten Steinhoff

Germanistisches Seminar, Universität Siegen, Hölderlinstr. 3, 57068 Siegen, Deutschland

E-Mail: steinhoff@germanistik.uni-siegen.de
} 


\title{
Can monolingual interventions be effective across languages? How the language of schooling can support heritage languages
}

\begin{abstract}
In addition to contributing to the development of heritage language (HL) and plurilingual skills amongst HL speakers, one of the goals of HL classes is often (mis)construed as their ability to promote the development of the language of schooling. For the overwhelming majority of HL speaking students in Germany, however, such an assumption seems difficult. These students have completed their entire schooling in German and thus have more highly developed literal skills in German than in the HL. The project reported here took this situation into account. In a longitudinal study carried out in sixth-grade classes, it investigated how differential writing interventions in the majority language German might affect the quality of writing in the HL Turkish. As well, it investigated associations between the writing quality and the writing process in both languages. The results indicate that plurilingual competences in the writing of these students are at least partially characterized by transversality and thus not language-specific, but that they may be supported by a transfer of language knowledge from the majority language to the HL. We conclude that effective cross-language writing instruction should focus on such subskills that could be transversally relevant.
\end{abstract}

Keywords Heritage languages · Translingual writing $\cdot$ Revision $\cdot$ Plurilingualism · Interlingual transfer

\section{Einleitung}

Schüler*innen sollen im Deutschunterricht, Fremdsprachenunterricht (z. B. Englisch, Französisch, Latein) und Herkunftssprachenunterricht (z. B. Polnisch, Türkisch, Russisch) in einem relativ kurzen Zeitraum eine Vielzahl (bildungs-)sprachlicher Kompetenzen erwerben. Eine Möglichkeit, dieser Herausforderung zu begegnen, besteht in der Entwicklung sprachenübergreifender didaktischer Konzepte. Aus theoretischer Sicht spricht vieles dafür, dabei von derjenigen Sprache auszugehen, in der die Schüler*innen am kompetentesten sind, und Teilkompetenzen $\mathrm{zu}$ fokussieren, die sie in mehreren Sprachen benötigen. Bislang liegen allerdings kaum empirische Studien zur Wirksamkeit eines solchen Vorgehens vor.

Auf dieses Desiderat wurde am Beispiel der Förderung von Schreibkompetenzen mit einem Projekt reagiert, das vom BMBF im Rahmen des Forschungsschwerpunkts „Sprachliche Bildung und Mehrsprachigkeit“" gefördert wurde. Es bezog sich auf das Schreiben in der Unterrichtssprache Deutsch und der Herkunftssprache Türkisch. Die im vorliegenden Beitrag berichteten Teilergebnisse der Studie beziehen sich auf die Frage, wie sich sprachliche Hilfen, die im Deutschunterricht gegeben wurden, auf die Qualität türkischer Schülertexte, die im Türkischunterricht erhoben wurden, auswirkten, und die Frage, welche Zusammenhänge zwischen der Qualität deutscher und türkischer Schülertexte sowie zwischen den Revisionshandlungen, die in den beiden Sprachen realisiert wurden, bestanden (zu intralingualen Zusammenhängen vgl. Rüßmann 2018). Dazu wurde eine Interventionsstudie in elf 6. Klassen 
durchgeführt. Die Ergebnisse zeigen, dass Schüler*innen unter geeigneten Interventionsbedingungen ihre im Deutschen erworbenen Schreibkompetenzen auch beim Schreiben im Türkischen nutzen können. Zudem zeigten sich signifikante interlinguale Zusammenhänge zwischen den beiden Sprachen hinsichtlich der Textqualität und der Art und Anzahl der Revisionshandlungen. Dies deutet darauf hin, dass wesentliche Facetten von Schreibkompetenzen sprachenübergreifend sind und diese Kompetenzen interlingual gefördert werden können.

In Abschn. 2 wird der theoretisch-empirische Rahmen skizziert und in den Abschn. 3 bis 5 die Interventionsstudie vorgestellt. In Abschn. 5 werden ausgewählte Ergebnisse referiert, die in Abschn. 6 diskutiert werden.

\section{Interlingualer Transfer und interlinguale Transversalität von Schreibkompetenzen}

Der Sprachenunterricht an deutschen Schulen folgt einem ,multiplen Monolingualismus": Für den Deutsch-, Fremdsprachen- und Herkunftssprachenunterricht gibt es jeweils eigene Fächer, Curricula und Lehrwerke (vgl. Marx 2014). Im Widerspruch dazu stehen empirische Ergebnisse, die eindeutig nachweisen, dass einzelsprachliche Kompetenzen von Bilingualen (zweisprachigen Individuen) und Plurilingualen (mehrsprachigen Individuen) interlingual (sprachenübergreifend) positiv zusammenhängen (vgl. zusammenfassend Singleton und Aronin 2019). Eine Vielzahl von Studien mit unterschiedlichsten Sprachenkonstellationen, aus unterschiedlichsten Nationen und mit unterschiedlichsten Lernenden weist nach, dass dies auch und gerade für Lese- und Schreibkompetenzen in der Schule und Universität gilt (vgl. Cummins 2000).

Wie solche Zusammenhänge entstehen, ist umstritten. Zu vermuten ist, dass bestimmte (Teil-) Kompetenzen zwar sprachenspezifisch erworben werden, dann aber auch in anderen Sprachen zur Verfügung stehen. Dabei handelt es sich nicht um einen schlichten (Aus-) Tausch oder eine Weitergabe von Wissen bzw. Kompetenzen, wie die frühe Interferenzforschung anhand von Fehleranalysen zu belegen versuchte (u.v.a. schon Juhasz 1970). Vielmehr werden die jeweiligen Kompetenzen durch einen Austauschprozess für andere Kontexte geöffnet, der gemeinhin als Transfer bezeichnet wird (z. B. Odlin 1989). Dies kann darin resultieren, dass bestimmtes Wissen bzw. bestimmte Kompetenzen in mehreren Sprachen zur Verfügung stehen, d.h. transversal werden; solche transversalen Kompetenzen sind für unterschiedliche Dimensionen des Sprachgebrauchs nachgewiesen (vgl. Berthele und Lambelet 2018).

Die (schulische) Instruktion kann dabei eine kleinere oder größere Rolle spielen. Dass sprachliche Kompetenzen ohne gezielten Unterricht unter gewissen Bedingungen transversal werden können, ist der Grundgedanke der verbreiteten ,Interdependenzhypothese“ (engl. interdependence principle) von Cummins (zuerst 1981): „To the extent that instruction in Lx is effective in promoting proficiency in Lx, transfer of this proficiency to Ly will occur provided there is adequate exposure to Ly and adequate motivation to learn Ly“ (Cummins 2010, S. 15). Dass relevante Kompetenzen durch eine von außen (durch die Lehrkraft oder Lehr-/Lernmaterialien) gesteuerte 
lernerseitige Reflexion der Plurilingualität (also der individuellen Mehrsprachigkeit) gefördert werden können, ist der Grundgedanke des Mainstreams der Mehrsprachigkeitsdidaktik. Diese Annahme geht insofern deutlich über den Grundsatz der Interdependenzhypothese von Cummins hinaus.

Wenn es in Deutschland um die Entwicklung der lebensweltlichen Mehrsprachigkeit geht, wird i.d.R. die Position eingenommen, dass ein didaktisch geförderter Transfer von Fähigkeiten von einer Sprache in eine andere wünschenswert sei. Dabei wird insbesondere gefordert, dass Lerner*innen mit nichtdeutschen Familiensprachen ihre herkunftssprachlichen Kompetenzen für die Entwicklung der schulisch relevanten Deutschkompetenzen nutzen (können) sollten. Der herkunftssprachliche Unterricht wird mithin als Steigbügelhalter für den amtssprachlichen Unterricht aufgefasst (Baker 2011, S. 207 und S. 209 ff.). Wirklich konsequent war man hier allerdings nie. Denn das schulische Curriculum ist in Deutschland nach wie vor ein Einzelsprachencurriculum; es führt also nicht vom herkunftssprachlichen Unterricht zum Deutschunterricht.

Ein solches curriculares Vorgehen ist allerdings auch nicht unbedingt ratsam. Denn die schulisch relevanten Deutschkompetenzen sind vor allem bildungssprachliche Kompetenzen, also Lese- und Schreibkompetenzen sowie konzeptionell schriftlich geprägte mündliche Sprachkompetenzen. So gesehen ist die SteigbügelhalterAnnahme eigentlich nur dann plausibel, wenn die Lerner*innen zuvor nur wenig Kontakt mit deutschen Texten hatten, z. B. dann, wenn sie im Schulalter als sogenannte „Seiteneinsteiger*innen“ neu zugewandert sind oder als Ausländer*innen in Deutschland ein Studium aufnehmen. In solchen Fällen können die bildungssprachlichen Kompetenzen, die in der Herkunftssprache erworben worden sind, den Erwerb bildungssprachlicher Kompetenzen im Deutschen stützen. Weniger plausibel ist die Steigbügelhalter-Annahme hingegen, wenn die Lerner*innen in Deutschland eingeschult und in der deutschen Sprache erstliteralisiert worden sind. Das ist insbesondere dann der Fall, wenn sie die Sekundarstufe I besuchen. Dann verfügen sie im Deutschen i.d.R. über höhere bildungssprachliche Kompetenzen als in ihren Herkunftssprachen. Dies lässt sich z. B. daran ablesen, dass sie in ihren Herkunftssprachen oft nur schwache Lesekompetenzen ausbilden (vgl. Brehmer und Mehlhorn 2015). Diese Sprachen nutzen sie vorwiegend für alltagssprachliche Zwecke, etwa den Austausch mit der Familie oder Freunden.

Daraus lässt sich der folgende Schluss ziehen: Will man in Deutschland das bildungspolitische Ziel verfolgen, die gesellschaftliche Vielsprachigkeit und die individuelle Plurilingualität so zu fördern, dass Schüler*innen neben der schulischen Majoritätssprache in mindestens zwei Sprachen bildungssprachliche Kompetenzen auf einem möglichst hohen Niveau erreichen (vgl. z. B. Rat der Europäischen Union 2015), sollte man dort ansetzen, wo die meisten Schüler*innen über die größten bildungssprachlichen Ressourcen verfügen - im Deutschen - und sich darum bemühen, diese Ressourcen auch für andere Sprachen nutzbar zu machen.

Vor diesem Hintergrund überrascht es, dass sich die Schreib- und Mehrsprachigkeitsforschung national wie international bislang kaum mit der Förderung plurilingualer Schreibkompetenzen bei Schüler*innen mit Herkunftssprachen auseinandergesetzt hat (vgl. Marx 2017). Sie konzentriert sich vielmehr auf Lernertexte, die in der schulischen Majoritätssprache verfasst werden, vergleicht diesbezüglich auto- 
chthone Lerner*innen mit Lerner*innen mit einer „Zweitsprache“ bzw. einem „Migrationshintergrund" und konzentriert sich dabei auf orthographische, grammatische, lexikalische und allgemeine Defizite in den Texten letzterer. Die wenigen Studien, die mindestens zwei Sprachen derselben Lernenden untersuchen, sind selten und fokussieren nicht die interlinguale Förderung, sondern den Kompetenzvergleich (vgl. zusammenfassend Reichert und Marx 2020).

Für die transversale Öffnung von (Schreib-)Kompetenzen bietet sich zunächst, von der Interdependenzhypothese ausgehend, eine lernförderliche Inbezugsetzung von Aspekten des Kontextes, der Kultur und der Kognition an, die für mehrere Sprachen relevant sind. Damit ist gemeint, dass für Lerner*innen Sprachgebrauchskontexte geschaffen werden sollten, die auf bestimmte Funktionen ausgerichtet sind (z.B. Überzeugen), und ihnen eine Orientierung an kulturellen Mustern des Sprachgebrauchs (z. B. Argumentieren) und eine Inanspruchnahme und Erweiterung ihres Wissens (z. B. Fach- und Sprachwissen) ermöglichen.

Dazu kann beispielsweise an das deutschdidaktische Konzept der „Schreibarrangements“ angeschlossen werden (vgl. Steinhoff 2018). Schreibarrangements sind komplexe Schreiblernaufgaben. Sie weisen eine Reihe von Merkmalen auf, die nach Ergebnissen der Schreibforschung für den Schreibunterricht bedeutsam sind, z. B. eine relevante didaktische Textsorte (,Textform“), eine*n dazu passende Leser*in (,Adressat“) und zielführende Hilfen zur Formulierung des Textes (,Sprache“). Dass Schreibarrangements monolingual wirksam sind, hat Anskeit (vgl. 2019) in einer umfangreichen Interventionsstudie in der 4. Klasse nachgewiesen. Sie konnte zeigen, dass Schreibaufgaben, die alle Merkmale von Schreibarrangements erfüllten, zu besseren Texten führten als Schreibarrangements, die nur einen Teil dieser Merkmale erfüllten. Die konkrete Ausgestaltung eines Schreibarrangements kann in Anlehnung an Bachmann und Becker-Mrotzek (2010) als „Profilierung“ bezeichnet werden.

Ein potenzieller Nutzen von Schreibarrangements für die Förderung bi- und plurilingualer Kompetenzen betrifft die Merkmale Textform und Sprache. Textformen können sprachenübergreifend ähnlich konzipiert werden und durch die damit einhergehenden inhaltlichen und strukturellen Gemeinsamkeiten lernerseitig sprachenübergreifende Lernprozesse anbahnen. Ein vergleichbares Potenzial hat das Merkmal Sprache, insbesondere dann, wenn ,Textprozeduren“ eingesetzt werden (vgl. Feilke 2014). Textprozeduren sind textsortentypische Formulierungsmuster. Studien zur Entwicklung von Schreibkompetenzen zeigen, dass der kompetente Gebrauch dieser Formulierungsmuster mit einer vergleichsweise hohen Textqualität in Zusammenhang steht (vgl. Feilke 2014). Textprozeduren bestehen aus ,Textprozedurenausdrücken“ (kurz: „Ausdrücke“, z. B. „A sieht aus wie B“, „A B’ye benziyor“, „A looks like B“) und „Textprozedurenschemata“ (kurz: „Schemata“, z. B. Vergleichen). Die Ausdrücke haben ein sprachenspezifisches Gepräge, die Schemata sind dagegen in verschiedenen Sprachen gebräuchlich und daher für interlinguale Erwerbsprozesse offen.

Genau das macht Textprozeduren zu potenziellen ,Boostern“ der Förderung von Schreibkompetenzen in verschiedenen Sprachenfächern. Um sie so in das Curriculum zu integrieren, dass sie die zeitlichen Kapazitäten der Sprachenfächer nicht übersteigen, und um außerdem das Potenzial der Sprache zu nutzen, in der die meisten Schüler*innen am besten schreiben, bietet es sich an, vom Deutschunter- 
richt auszugehen. Dies resultiert in der im Weiteren behandelten Forschungsfrage, wie sich monolinguale, aber zugleich transformationsoffene Schreibarrangements, die Textprozeduren fokussieren, auf das Schreiben in einer anderen Sprache auswirken. Unsere Studie folgt mithin einem didaktischen Konzept, das nicht vom oben beschriebenen Grundgedanken der Mehrsprachigkeitsdidaktik, sondern von der Interdependenzhypothese ausgeht.

Textprozeduren können zur Bildungssprache gerechnet werden und kommen sowohl in der schriftlichen als auch in der (durch Schriftlichkeit geprägten) mündlichen Kommunikation vor. Dass in unserer Studie die produktive, schriftliche Sprachkompetenz des Schreibens untersucht wird, gründet einerseits darin, dass diese Kompetenz für die sprachliche Verständigung zentral ist, aber im Herkunftssprachenunterricht oft vernachlässigt wird. Andererseits gründet sie darin, dass das Potenzial einer Didaktisierung von Textprozeduren für das sprachenübergreifende Lernen hier - an authentischen Texten - besonders gut exemplarisch beobachtet werden kann.

Für die didaktische Nutzung von Textprozeduren bietet sich eine Integration in den Schreibprozess an. Diese Einsicht beruht auf der Beobachtung, dass Textprozeduren das Formulieren stützen und vorantreiben (z. B. eine Gliederungsprozedur wie „erstens ... zweitens ... drittens ...“), und auf der Annahme, dass Schreibprozesskompetenzen ein transversales Gepräge haben. Unklar ist jedoch, welche Komponente(n) von Textprozeduren - Schema und Ausdruck oder nur Schema oder nur Ausdruck welches Förderpotential besitzt/besitzen. Unklar ist außerdem, ob die potenzielle Transversalität von Textprozeduren beide Komponenten betrifft oder - weil Ausdrücke sprachengebunden sind - nur die Schemata. In unserer Studie wird diesen Fragen nachgegangen und dafür das für das Schreiben in allen Sprachen maßgebliche Revidieren in den Blick genommen, d.h. das Überarbeiten eines Textes. Es gibt Indizien, dass Lernende - zumindest beim Schreiben in der Fremdsprache und der Erstsprache - ähnliche und ähnlich viele Revisionshandlungen auf ähnlichen sprachstrukturellen Ebenen (Wort, Phrase, Satz) verwenden (u.a. Stevenson et al. 2006; zusammenfassend s. Reichert und Marx 2020). Das Revidieren scheint mithin Teil einer transversalen Schreibkompetenz zu sein, was nahelegt, diese Kompetenz i. S. der Interdependenzhypothese interlingual zu fördern.

\section{Forschungsfragen und Hypothesen}

Nachfolgend werden drei ausgewählte, für den vorliegenden Aufsatz relevante Forschungsfragen des Projekts zu interlingualen Zusammenhängen genannt (für Details vgl. Wenk 2018):

1. Welche Auswirkungen hat die Textqualität im Deutschen, die durch den wiederholten Einsatz textprozedural unterschiedlich profilierter Schreibarrangements (keine sprachlichen Hilfen oder Hilfen zu Ausdrücken oder Hilfen zu Schemata oder Hilfen zu Ausdrücken und Schemata) im Deutschen beeinflusst wird, auf die Textqualität im Türkischen? (Kausalitätsfrage über eine Mediatorvariable)

2. Bestehen Zusammenhänge bezüglich der Textqualität im Deutschen und Türkischen? (Zusammenhangsfrage zu Produktmerkmalen) 
3. Bestehen Zusammenhänge bezüglich der Art und Anzahl der Revisionshandlungen zwischen dem Deutschen und Türkischen? (Zusammenhangsfrage zu Prozessmerkmalen)

Die Hypothesen beruhen auf den in Abschn. 2 skizzierten Annahmen und lauten:

- ad 1: Schema- und prozedurprofilierte Schreibarrangements im Deutschen führen über die Wirkung auf deutschsprachige Texte zu besseren Texten im Türkischen als ausdrucks- und basisprofilierte Schreibarrangements.

- ad 2 und 3: Es bestehen interlinguale Zusammenhänge zwischen der Textqualität im Deutschen und im Türkischen und den Revisionshandlungen in diesen Sprachen.

\section{Stichprobe}

Im Rahmen der Studie wurden zwischen Dezember 2014 und April 2015 Text-, Fragebogen- und Testdaten in elf 6. Klassen einer Gesamtschule $(n=32)$ sowie einem Gymnasium $(n=59)$ in Nordrhein-Westfalen erhoben. Die für diesen Beitrag relevante Teilstichprobe besteht aus 91 bilingualen Schüler*innen (weiblich $n=53$, männlich $n=26$, fehlende Angaben $n=12$ ), die sowohl am Deutschunterricht (,DU“) als auch am Türkischunterricht (,TU“) teilnahmen. Alle Schüler*innen waren in Deutschland geboren und hatten ihre gesamte bisherige Schullaufbahn dort absolviert. Zudem sprachen alle Teilnehmer*innen in ihren Familien (auch) Türkisch und besuchten im Rahmen des schulischen Sprachenangebots pro Woche drei Unterrichtseinheiten Türkisch. Im Türkischlesekompetenztest (s. unten) erreichten vier Teilnehmer*innen einen Wert auf oder unter dem GER-Niveau A1, 69 Teilnehmer*innen einen Wert auf oder unter dem GER-Niveau A2 (aber über A1) und 18 Teilnehmer*innen einen Wert auf oder unter dem GER-Niveau B1 (aber über A2). Die Lesekompetenzen dieser Schüler*innen waren also recht schwach und lagen deutlich unter dem Wert ihrer Deutschlesekompetenzen; letztere entsprachen dem in der jeweiligen Schulform erwartbaren Niveau (vgl. Marx und Steinhoff 2017b).

Da für die Analyse der interlingualen Zusammenhänge bei der Art und Anzahl der Revisionshandlungen (Forschungsfrage 3) das Ergebnis zur Forschungsfrage 1 berücksichtigt werden musste, war es erforderlich, die Stichprobe einzugrenzen. Denn die verschiedenen Schreibarrangements im Deutschunterricht wirkten sich unterschiedlich auf die Qualität der deutschen und dadurch anschließend auch der türkischen Texte aus und stellten deshalb einen möglichen Störfaktor für die Untersuchung der Revisionshandlungen der Experimentalgruppen dar. Aus diesem Grund konnte allein die Kontrollgruppe, deren Überarbeitungen nicht durch die Intervention beeinflusst wurden, berücksichtigt werden. Des Weiteren konnten bei der Untersuchung der Forschungsfrage 3 nicht alle Messzeitpunkte (MZP) berücksichtigt werden. Da die Textqualität in beiden Sprachen zum MZP 3 anstieg und sich dann stabilisierte und sich im Türkischen zum MZP 5 aus unbekannten Gründen einen „Knick“ bei zwei Interventionsgruppen zeigte, wurden nur die robusteren Werte der MZP 3 und 4 für beide Sprachen einbezogen. Damit ergab sich für die Forschungs- 
frage 3 eine relativ kleine Teilstichprobe von $n=22$ Schüler*innen mit insgesamt 42 deutschen und 38 türkischen Texten (deskriptive Statistik s. unten). Durch die hohe Anzahl der durchgeführten Analysen war die Güte der statistischen Analysen gleichwohl nicht beeinträchtigt.

Für die Intervention wurden vier Interventionsgruppen im Deutschunterricht gebildet (s. unten). In den elf involvierten Klassen wurden die Schüler*innen nach der Qualität ihres ersten, im Prätest unter der Kontrollkondition verfassten deutschsprachigen Textes balanciert. Bei der Bildung der Versuchsgruppen wurde auf weitere potentiale Störfaktoren (z. B. Geschlecht) geachtet.

\section{Methode}

\subsection{Schreibarrangements im Deutsch- und im Türkischunterricht}

Die Studie untersuchte die Textproduktion prozess- und produktbezogen: hinsichtlich der Revisionshandlungen und der Textqualität. Für die Didaktisierung und die Erhebung der Daten wurden verschiedene materialbasierte Schreibarrangements entwickelt. In allen Schreibarrangements ging es um Figurenbeschreibungen: Die Schüler*innen hatten die Aufgabe, defizitäre Beschreibungen (,Ausgangstexte“) von Superheld*innen und Superschurk*innen zu überarbeiten. Ihre Texte sollten es einer Leserin bzw. einem Leser, die bzw. der diese Figuren nicht kennt, ermöglichen, sie sich vorzustellen. Es wurde jedoch zugleich darauf aufmerksam gemacht, dass die Leserin bzw. der Leser fiktiv ist und es eigentlich darum geht, Schreibaufgaben für ein Schulbuch zu testen, an dem die Projektleiter*innen arbeiteten. Diese „Coverstory“ sollte die Glaubwürdigkeit des Vorhabens erhöhen.

Mit der Beschreibung wurde eine Textform ausgewählt, die für die Sekundarstufe I zentral ist, und mit den Superheld*innen/-schurk*innen ein Thema festgelegt, das in diesem Alter sehr beliebt ist. Der Ausgangstext sollte den Schüler*innen einen groben Eindruck vom erwarteten Text vermitteln und durch seine offenkundigen Defizite (als ,Provokation“) vor Augen führen, dass er dringend revidiert werden muss. Der Fokus der Textproduktion in den Schreibarrangements lag mithin auf der Revision, einer Tätigkeit, die in der Schreibforschung für den Erwerb von Schreibkompetenzen als zentral gilt, im schulischen Alltag aber nach wie vor selten geübt wird.

Ausgehend von Erkenntnissen zum Erwerb schriftlicher Beschreibungskompetenzen in der Sekundarstufe I wurden für die Schreibarrangements insgesamt acht Textprozeduren fokussiert und ausgewertet:

1. „Kategorisieren“ (die Figur vorstellen),

2. „Kontextuieren“ (die Umgebung der Figur beschreiben),

3. ,Vergleichen“ (die Figur mit Dingen und Personen in Verbindung bringen),

4. „Strukturieren“ (die Figur in einer guten Reihenfolge beschreiben),

5. „Visualisieren“ (den Körper, die Kleidung und die Supergegenstände der Figur beschreiben),

6. „Verorten“ (den Platz der Supergegenstände beschreiben), 
Tab. 1 Schreibarrangement am Beispiel der Schemaprofilierung zum Superhelden „Rocket Rob“ und zur Superheldin „Rüzgarli Rana“ (,Stürmische Rana“)

\section{Deutschunterricht: Schritt 1}

Lies dir die Beschreibung von Rocket Rob genau durch. Kannst du dir Rocket Rob mit dieser Beschreibung gut vorstellen?

Rocket Rob, der Superheld. Da sind Häuser. Rocket Rob ähnelt einem Menschen. Er hat Arme. Ganz unten: die Füße. Er hat da eine Pizza. Er fliegt. Dafür gibt es Gründe

\section{Deutschunterricht: Schritt 3 (Beispiel: Sche- maprofilierung)}

Damit sich dein Leser Rocket Rob gut vorstellen kann, solltest du deine Beschreibung sprachlich gut aufbauen. Lies dir dafür die Schreibtipps genau durch.

- Stelle Rocket Rob vor

- Beschreibe Rocket Robs Umgebung.

- Vergleiche Rocket Rob mit Dingen und Personen.

- Beschreibe Rocket Rob in einer guten Reihenfolge (z. B. von oben nach unten, vom Auffälligen zum Unauffälligen).

- Beschreibe Rocket Robs Körper, Kleidung und Supergegenstände.

- Beschreibe den Platz der Supergegenstände von Rocket Rob.

- Beschreibe, was Rocket Rob womit macht.

- Beschreibe, warum Rocket Rob das alles so tut

\section{Deutschunterricht: Schritt 2}

Lies dir die Beschreibung von Rocket Rob genau durch. Kannst du dir Rocket Rob mit dieser Beschreibung gut vorstellen?

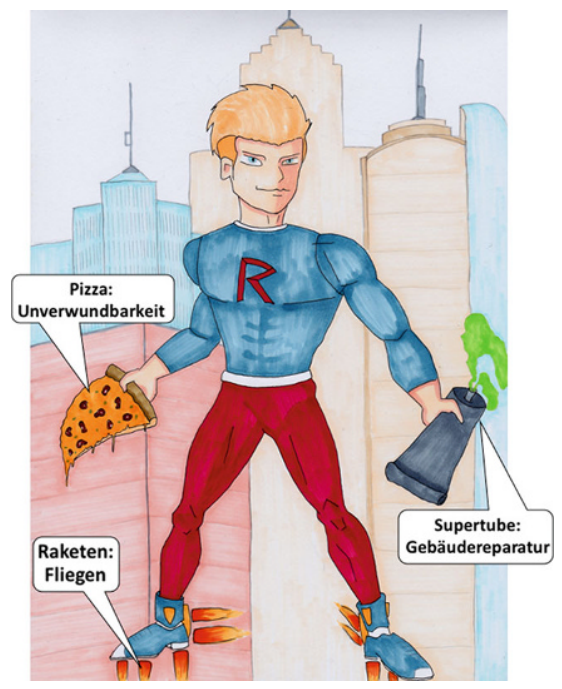

\section{Deutschunterricht: Schritt 4}

Schreibe jetzt eine gute Beschreibung von Rocket Rob. Vergiss nicht, dass dein Leser Rocket Rob nicht kennt! Mit deiner Beschreibung soll er sich Rocket Rob genau vorstellen können! 
Tab. 1 (Fortsetzung)

\section{Türkischunterricht: Schritt 1}

Rüzgarlı Rana betimlemesini dikkatlice oku! Sen bu betimlemeden Rüzgarlı Rana'yı hayal edebiliyor musun?

Rüzgarlı Rana, iyi kahraman. Evler var. Rüzgarl Rana bir insan gibi. Onun saçları, ă̆zı ve burnu var. Altında: ayakkabılar. Şurada Rüzgarlı Rana'nın bisküvi kutusu var. Rüzgarlı Rana uzun atlar. Onun için sebepler var

\section{Türkischunterricht: Schritt 2}

Rüzgarlı Rana betimlemesini dikkatlice oku! Sen bu betimlemeden Rüzgarlı Rana'yı hayal edebiliyor musun?

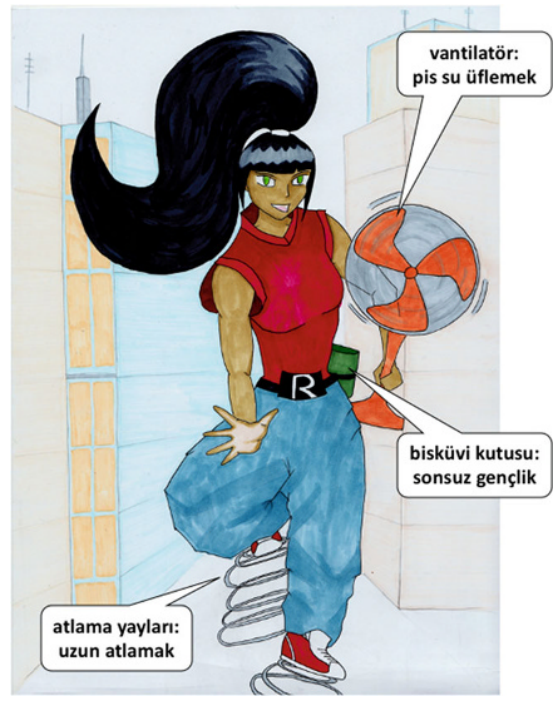

Türkischunterricht: Schritt 3

Düzgün bir Rüzgarlı Rana betimlemesi yaz. Unutma ki, senin okuyucun Rüzgarlı Rana'yı tanımıyor ve senin betimlemenle Rüzgarlı Rana’yı doğru hayal edecek!

Im TU wurden die Schritte wie im DU formuliert - bis auf die Intervention, die dort entfiel

7. „Agentivieren“ (die Handlungsinstrumente und Handlungen der Figur beschreiben),

8. „Deuten“ (die Intentionen der Figur beschreiben).

Der Ausgangstext bestand aus acht Sätzen, in denen je eine Textprozedur - defizitär - realisiert wurde. Die Schüler*innen wurden mit diesem Text also implizit auf die Textprozeduren aufmerksam gemacht. Die explizite Vermittlung der Textprozeduren hingegen war Gegenstand der Intervention (s. Abschn. 5.2). Darüber hinaus waren die Textprozeduren für die Einschätzung der Textqualität maßgeblich (s. Abschn. 5.4).

Alle Schreibarrangements waren gleich aufgebaut. Dies wird in Tab. 1 am Beispiel der „Schemaprofilierung“ (s. unten) veranschaulicht. Im ersten Schritt lasen die Schüler*innen den Ausgangstext zur Figur und sollten überlegen, ob sie sich die Figur auf dieser Grundlage gut vorstellen konnten. Im zweiten Schritt betrachteten sie eine Zeichnung der Figur und sollten diese mit dem Ausgangstext abgleichen. Teil der Zeichnung waren „Textfelder“, in denen Informationen zur Figur gegeben wurden, die über das visuell Darstellbare hinausgingen (,Supergegenstände“ 
und „Superkräfte“). Im dritten Schritt fand die Intervention statt: Die Interventionsgrupppen erhielten unterschiedliche textprozedurale Hilfen. Und im vierten und letzten Schritt revidierten die Schüler*innen den Ausgangstext.

\subsection{Interventionsphase im Deutschunterricht}

Für die Interventionsphase im DU (MZP 2 bis 5) wurden drei Interventionsgruppen (kurz: „IG“) und eine Kontrollgruppe (kurz: „,KG“) gebildet. Diese Gruppen und die entsprechenden Schreibarrangements unterschieden sich allein hinsichtlich der acht oben genannten Textprozeduren. In den Interventionsgruppen wurden Hilfen zu Textprozeduren in unterschiedlich didaktisierter Form listenförmig angeboten (,textprozedurale Profilierung"). Dabei wurde von den in Abschn. 2 erläuterten Komponenten ausgegangen, dem sprachenspezifischen „Ausdruck“ und dem sprachenübergreifenden „Schema“. In einer IG („Prozedurprofilierung“) wurden Ausdruck und Schema zusammen angeboten, in den beiden anderen IGs wurden, um eventuellen differentiellen Effekten nachzugehen, nur Ausdrücke oder nur Schemata angeboten. In der Kontrollgruppe wurden keine textprozeduralen Hilfen angeboten.

1. IG „Ausdrucksprofilierung“ (kurz: „AP“): nur Ausdruckshilfen (z. B. ,,.. bei ... handelt es sich um ...", ,... ist kein ... sondern ...")

2. IG „Schemaprofilierung“ (kurz: „SP“): nur Schemahilfen (z. B. „Stelle Rocket Rob vor.")

3. IG „Prozedurprofilierung“ (kurz: „PP“): Kombinationen von Ausdrucks- und Schemahilfen (z.B. „Stelle Rocket Rob vor. Dafür kannst du z.B. schreiben: ... bei ... handelt es sich um ..., ... ist kein ... sondern ...")

4. KG „Kontrollgruppe“: „Basisprofilierung“ (kurz: „BP“) ohne textprozedurale Hilfen

Das Gruppendesign lässt einen wesentlichen Unterschied zu Interventionsstudien erkennen, die in der Sprachdidaktik üblich sind (vgl. Marx und Steinhoff 2017a, S. $258 \mathrm{ff}$.). Die Interventionsgruppen wurden nicht mit regulärem Unterricht verglichen, sondern mit einer Kontrollgruppe, die, von den textprozeduralen Hilfen abgesehen, den gleichen Unterricht in den gleichen Klassen wie die Interventionsgruppen erhielt. Dieser Unterricht erfüllte wesentliche Merkmale von Schreibarrangements und unterstützte den Schreibprozess der Schüler*innen mithin nach neuesten Erkenntnissen der Schreibforschung. So wurde gewährleistet, dass die Ergebnisse tatsächlich auf die Intervention zurückgeführt werden können - und nicht z. B. auf die Neu- und Besonderheit des Schreibarrangements oder die Lehrperson.

\subsection{Studiendesign}

Die Daten wurden zwischen Dezember 2014 und April 2015 erhoben. Es gab 14 MZP: sieben im DU (Prätest [ausschließlich Basisprofilierung] - vier Interventionen [alle Profilierungen] - zwei Posttests [ausschließlich Basisprofilierung]) und sieben im TU (ausschließlich Basisprofilierung). Zwischen dem Prätest (DU) bzw. Erhebung 1 (TU) und der Intervention 1 (DU) bzw. Erhebung 2 (TU) lagen zwei Monate. Die Interventionen 1 bis 4 (DU) bzw. die Erhebungen 2 bis 5 (TU) fanden 
in einwöchigem Abstand statt. Es folgten im Abstand von einer und sechs Wochen die Posttests 1 und 2 (DU) bzw. die Erhebungen 6 und 7 (TU). Die Erhebungen im TU erfolgten stets in der gleichen Woche wie die Erhebungen im DU. Zu jedem MZP schrieben die Schüler*innen einen Text zu einer jeweils neuen Superfigur. Da es im DU und TU insgesamt 14 MZP gab, wurden also 14 Superheld*innen und -schurk*innen eingesetzt. Dabei wurde auf eine vergleichbare Komplexität geachtet (z.B. gleiche Anzahl an Kleidungsstücken).

Die Schüler*innen widmeten sich zu allen MZP der in Abschn. 5.1 erläuterten Aufgabe: Sie überarbeiteten defizitäre Beschreibungen von Superheld*innen und -schurk*innen. Der Prätest diente der Balancierung: Die dort erhobenen Schülertexte wurden global auf ihre Qualität geprüft und die Qualitätsurteile dann genutzt, um in jeder Klasse vier vergleichbar leistungsstarke Gruppen zu bilden. Die Schüler*innen wurden im DU zu allen MZP der gleichen Untersuchungsgruppe zugeordnet. Im TU partizipierten alle Schüler*innen an der Basisprofilierung (Kontrollbedingung).

Tab. 2 zeigt die Datenerhebung im Überblick:

Die Datenerhebung fand im Klassenverbund statt. Der Ablauf war bei allen Messzeitpunkten bis auf die Interventionsmaßnahme gleich: Die Schüler*innen wurden von der bzw. dem Versuchsleiter*in auf der Grundlage eines teilstandardisierten Leitfadens an den Schreibanlass erinnert und widmeten sich dann rein materialbasiert und in Einzelarbeit mit Stift und Papier dem Schreibarrangement. Zu Beginn der MZP 1 und 2 wurden überdies sowohl im DU als auch im TU Erklärvideos zum Überarbeiten gezeigt. In diesen zwei kurzen Videos, eines in deutscher, eines in türkischer Sprache, erklärt ein*e erwachsene*r Schreiber*in am Beispiel einer Zimmerbeschreibung in leicht zugänglicher Sprache, wie man einen Text überarbeitet. Damit wurde auf die während der Pilotierung gemachte Beobachtung reagiert, dass den Schüler*innen das Überarbeiten weitgehend unbekannt war, und ein weiterer Schreibmotivationsimpuls gesetzt. Die Videos wurden sowohl im DU als auch im TU direkt vor den beiden ersten Erhebungen gezeigt.

Um die Balancierung in den jeweiligen Gruppen zu prüfen sowie relevante Kontrollvariablen (Kovariaten) zu erheben, wurden zwischen den MZP 1 und 2 individuelle Merkmale und die Deutsch- sowie die Türkisch-Lesekompetenzen der Schüler*innen erhoben. Dazu wurden ein umfangreicher Schülerfragebogen, u.a. zu Familiensprache(n) und Literalitätserfahrungen (vgl. Rüßmann 2018, S. $126 \mathrm{ff}$. sowie Wenk 2018, S. 127 ff.), der Frankfurter Leseverständnistest 5-6 (FLVT) (vgl. Souvignier et al. 2008) und, in Ermangelung eines relevanten standardisierten Tests, ausgewählte Leseaufgaben der in zahlreichen Schulen eingesetzten telc-Tests für die GeR-Niveaus A1, A2 und B1 für Türkisch eingesetzt (vgl. telc o.J.). Die Mit-

Tab. 2 Datenerhebungsdesign

\begin{tabular}{llllllll}
\hline & MZP 1 & MZP 2 & MZP 3 & MZP 4 & MZP 5 & MZP 6 & MZP 7 \\
& Woche 0 & Woche 1 & Woche 2 & Woche 3 & Woche 4 & Woche 5 & Woche 10 \\
\hline DU & DU1: & DU2: & DU3: & DU4: & DU5: & DU6: & DU7: \\
& BP & BP, AP, & BP, AP, & BP, AP, & BP, AP, & BP & BP \\
& & SP, PP & SP, PP & SP, PP & SP, PP & & \\
$T$ & TU1: & TU2: & TU3: & TU4: & TU5: & TU6: & TU7: \\
& BP & BP & BP & BP & BP & BP & BP \\
\hline
\end{tabular}


telwertsunterschiede an allen diesen Testvariablen unterschieden sich zwischen den vier Untersuchungsgruppen nicht. Die Einzelergebnisse werden an anderer Stelle dargelegt (vgl. Marx und Steinhoff 2017b).

\subsection{Datenauswertung}

Der Erfolg von Fördermaßnahmen wird in der Schreibforschung i.d.R. am Schreibprodukt und vereinzelt am Schreibprozess festgemacht. Beim Schreibprodukt interessieren v. a. die Textqualität und die - oft mit höherer Qualität zunehmende Textlänge, beim Schreibprozess insbesondere Textrevisionen, d. h. über formale Korrekturen hinausgehende Überarbeitungen (vgl. Anskeit 2019).

Gegenstand der Analyse im Rahmen der Fragestellungen 1 und 2 sind 614 deutsche Texte und 607 türkische Texte, die von 91 bilingualen Schüler*innen stammen, die sowohl im DU als auch im TU an mindestens fünf Erhebungen teilnahmen. 11 weitere Teilnehmende entsprachen dieser Anforderung nicht und wurden deshalb ausgeschlossen. Sie stammten aus allen Versuchsgruppen und ihre Ergebnisse wichen von denen weiterer Schüler*innen nicht signifikant ab. Gegenstand der Analyse im Rahmen der Fragestellung 3 sind 42 deutsche und 38 türkische Texte, die von den 22 Schüler*innen stammen, die an der Basisprofilierung im DU teilnahmen (s. oben, Abschn. 4). Die Texte wurden pseudonymisiert und wortorthographisch korrigiert und dann bezüglich der Textqualität sowie der Art und Anzahl der Revisionshandlungen analysiert.

Um die Textqualität zu untersuchen, wurde ein analytisch-kriteriales Rating durchgeführt, das „Textprozedurenrating“. Es basiert auf der in Abschn. 2 erwähnten Erkenntnis, dass der angemessene Gebrauch von Textprozeduren in engem Zusammenhang mit einer hohen Textqualität steht. Das Textprozedurenrating wurde eigens für das Projekt entwickelt und ermöglichte es, die Texte in direkter Relation zu aufgabenspezifischen Erwartungen einzuschätzen. Die Entwicklung solcher Ratings ist ein Desiderat der Schreibforschung; dort wird die Textqualität bislang meist aufgabenunabhängig bestimmt. Das Textprozedurenrating bezog sich auf 7 der 8 in der Intervention eingesetzten Textprozedurenschemata: Kontextuieren, Vergleichen, Strukturieren, Visualisieren, Verorten, Agentivieren und Deuten (s. Abschn. 5.1). Das achte Textprozedurenschema, das Kategorisieren, wurde ausgeklammert, weil es bereits im Ausgangstext eine für den Nachweis der Textqualitätsentwicklung zu hohe Qualität aufwies und schon bei der ersten Erhebung zu entsprechenden Deckeneffekten führte. Pro Schema wurden, je nach Qualität der Umsetzung, 0 bis 4 Punkte auf einer Ordinalskala vergeben. Das Rating wurde von jeweils zwei unabhängigen, geschulten, Deutsch bzw. Türkisch sprechenden Rater*innen anhand eines Ratingmanuals durchgeführt und erzielte eine gute Interraterreliabilität von $\kappa=0,71 \mathrm{im}$ DU und $\kappa=0,79 \mathrm{im}$ TU.

Gegenstand der Analyse der Forschungsfrage 3 sind die Revisionshandlungen. Darunter verstehen wir Modifikationen von Sätzen aus dem Ausgangstext (sowohl im Deutschen als auch Türkischen). Für deren Analyse wurde das Textkorpus in MAXQDA eingepflegt, annotiert und kategorisiert. In Fortführung einschlägiger Klassifikationen von Revisionen (vgl. Fix 2000) wurden die entsprechenden Text- 
stellen direkt nach den folgenden fünf Typen von Revisionshandlungen bestimmt und analysiert (vgl. Marx und Steinhoff 2020, S. 13):

1. „Streichen“: Im Schülertext kommt ein Ausdrucksmuster aus dem Ausgangstext zur betreffenden Textprozedur nicht vor. (z. B. „Siyah Akba bir insan gibi.“; „Rocket Rob ähnelt einem Menschen.")

2. „Übernehmen“: Im Schülertext ist das Ausdrucksmuster mit dem Ausdrucksmuster im Ausgangstext identisch (z. B. ,Siyah Akba bir insan gibi.“; „Pollution Paul ähnelt einem Menschen.“").

3. „Verändern“: Im Schülertext ist das Ausdrucksmuster lexikalisch, morphologisch und/oder syntaktisch anders als im Ausgangstext und die Ausdrucksleerstellen sind inhaltlich ähnlich gefüllt (z.B. „Arkasında ağaçlar var.“; „Er sieht wie ein Mensch aus").

4. „Ersetzen“: Im Schülertext wird das Ausdrucksmuster aus dem Ausgangstext gestrichen und durch ein anderes Ausdrucksmuster des gleichen Textprozedurenschemas ersetzt (z.B. „Siyah Akba'nın arkasında büyük ağaçlar var.“; „Er ist in nem Wald." ).

5. „Erweitern“: Im Schülertext wird das Ausdrucksmuster aus dem Ausgangstext mindestens zweimal aufgegriffen. Diese Kategorie ergibt sich nur in Kombination mit Übernehmen/Verändern/Ersetzen (pro Textprozedur mehrfach möglich) (z. B. „Siyah Akba, kötü kahraman. [...] Siyah Akba bir oğlan vücudu ten rengi.“; „Pollution Paul ähnelt einem Menschen. [...] Er hat weiße Zähne wie ein Superheld“).

\subsection{Datenanalyse}

Bei der Beantwortung der Fragestellung 1 - welchen differentiellen Einfluss die Interventionen im Deutschunterricht auf die Textqualität im Türkischen über den Mediator Textqualität im Deutschen hatten -, waren mit der Datenanalyse spezifische Probleme verbunden. Diese hängen mit der Natur des Nachweises von interlingualen Transfereffekten auf Grund (wiederholter) didaktischer Interventionen zusammen und sind bis dato kaum zufriedenstellend gelöst worden (für eine Diskussion dieser Problematik sowie eine ausführlichere Darstellung des hier aufgeführten Lösungsvorschlags vgl. Marx 2020; frühere Lösungsversuche sind in Marx und Steinhoff 2017b; Wenk et al. 2016 und Wenk 2018 nachzulesen). Zusammengefasst besteht das Problem darin, dass der Effekt der Intervention im Deutschunterricht in seiner Wirkung auf die Textqualität im Deutschen berücksichtigt werden muss, um über diese Wirkung (den Mediator) seinen Effekt auf die Textqualität im Türkischen zu untersuchen. Um das Problem für die hier behandelte Studie zu lösen, wurden Mediationsanalysen (vgl. Hayes 2018) durchgeführt.

In Mediationsanalysen wird der Effekt der Intervention im Deutschen auf die Textqualität im Türkischen in zwei voneinander unabhängige Pfade aufgeteilt: (1) den direkten Pfad der Intervention auf die türkischen Texte ( $\left.c^{\prime}\right)$ sowie (2) den indirekten Pfad der Intervention auf die türkischen Texte, der über die Verbesserung in den deutschen Texten erfolgt. Statistisch erfasst wird dieser indirekte Wirkmechanismus der Intervention durch das Produkt der Pfade $a^{*} b(a=$ Effekt der Intervention auf deutsche Texte; $b=$ bereinigter Effekt dieses Mediators auf türkische Texte). Eine 


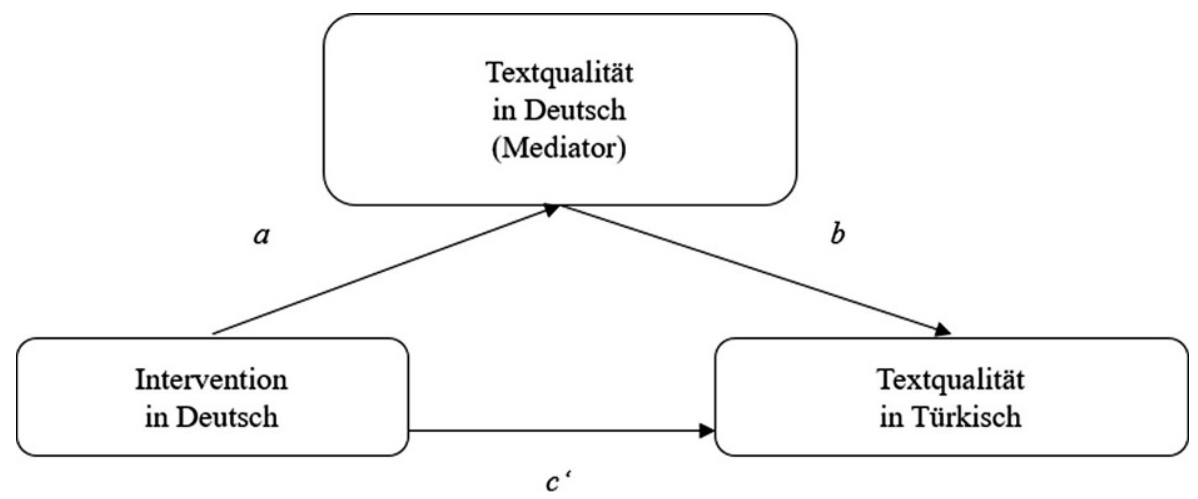

Abb. 1 Darstellung des Mediationspfads des indirekten Effekts von der Intervention im Deutschen auf die Textqualität im Türkischen $(a * b)$

inferenzstatistische Absicherung des Effekts erfolgt über Bootstrapping-Verfahren. Der Wirkmechanismus wird in einem einfachen Mediationsmodell wie in Abb. 1 dargestellt:

Für die Interpretation wesentlich ist, ob der indirekte Effekt des Mediators $(a * b)$ statistisch signifikant ist (s. Ergebnisdarstellung unten). Gemäß den Empfehlungen in Hayes (2018, S. 188) wurden separate Mediationsmodelle für jede der Interventionsgruppen mit Bezug zur Kontrollgruppe durchgeführt. 90\%-Konfidenzintervalle wurden auf Grund der explorativen Natur der Analysen gewählt.

Zur Beantwortung der Fragestellung 2 - ob ein Zusammenhang zwischen der Textqualität im Deutschen und Türkischen bestand - wurden die ordinalskalierten Rohwerte der Textqualität in beiden Sprachen zu den jeweils gleichen MZP mittels Spearman-Rangfolge-Korrelationen berechnet.

Zur Beantwortung der Fragestellung 3 - ob die Revisionshandlungen im Deutschen und Türkischen zusammenhingen - wurde mit dichotomen Daten gearbeitet. Das lag daran, dass pro Textprozedur in aller Regel nur eine Revisionshandlung vorgenommen wurde. Maßgeblich war, ob eine Revisionshandlung bei einer spezifischen Textprozedur vorkam (oder nicht). Die Analysen orientierten sich somit an der Auftretenswahrscheinlichkeit einer Revisionshandlung in den türkischen Texten in Abhängigkeit von der gewählten Revisionshandlung im Deutschen bei der gleichen Textprozedur. Die Analyse wurde für sechs der acht Textprozeduren durchgeführt; Visualisieren und Strukturieren wurden ausgeschlossen, da mit ihnen spezifische Auswertungsprobleme verbunden waren. (So wurden beim Visualisieren v.a. mehrere Gegenstände am Körper benannt, ohne dass sich die Handlung qualitativ änderte, während das Strukturieren v.a. auf Textebene stattfand und deshalb nicht hinreichend lokalisierbar war, vgl. Marx und Steinhoff 2020, S. 7). Anschließend wurden logistische Regressionen durchgeführt, mit denen das Vorkommen jeder der o.g. fünf Revisionshandlungen (Streichen, Übernehmen, Verändern, Ersetzen, Erweitern) in den türkischen Texten daraufhin geprüft wurde, ob die jeweilige Auftretenswahrscheinlichkeit durch die jeweilige Revisionshandlung im deutschen Text beeinflusst worden war. Die daraus resultierenden Odds-Ratios geben an, um wie viel die Wahrscheinlichkeit, dass eine Revisionshandlung vorkommt, durch das Vor- 
kommen dieser Variablen erhöht (wenn die Ratio über 1 liegt) oder reduziert (wenn die Ratio zwischen 0 und 1 liegt) wird oder aber gleich bleibt (wenn die Ratio bei 1 liegt) (vgl. Marx und Steinhoff 2020, S. 14).

\section{Ergebnisse}

\subsection{Effekte der Schreibarrangements im Deutschen auf die Textqualität im Türkischen}

Die durchgeführten Mediationsanalysen ergaben, dass bei keiner Interventionsgruppe statistisch signifikante, direkte Effekte ( $c$ '-Pfad) auf die Qualität der Türkischtexte $\mathrm{zu}$ verzeichnen waren (d.h. zwischen unteren und oberen Konfidenzintervallen lag der Wert 0). Dagegen gab es einen statistisch signifikant positiven, indirekten Effekt der Intervention im Deutschunterricht auf die Textqualität im Türkischen, der über den Mediator ging. Dieser Effekt gilt allerdings nur für zwei Interventionsgruppen. Die Schüler*innen der IG Schemaprofilierung, die von der Intervention im Deutschen profitierten, profitierten davon auch im Türkischen $(a * b=0,103)$. Das Gleiche galt für Schüler*innen der IG Prozedurprofilierung $(a * b=0,082)$. Die IG Ausdrucksprofilierung zeigte dagegen eine leicht gegenläufige Tendenz: Wer von dieser Intervention im Deutschen profitierte, war etwas schwächer im Türkischen $(a * b=-0,011)$. Da dieses Ergebnis allerdings nicht statistisch signifikant war, bleibt es hier unberücksichtigt. Die vollständigen Ergebnisse, inklusive der $a$ - und $b$-Pfade der Versuchsbedingungen, werden in Tab. 3 dargestellt.

Die Hypothese 1 bestätigt sich: Schema- und prozedurprofilierte Schreibarrangements im Deutschen führen zu signifikant besseren Texten im Türkischen als ausdrucks- und basisprofilierte Schreibarrangements, wenn die Voraussetzungen eines interlingualen Transfers i. S.d. Interdependenzhypothese (Verbesserung der Textqualität im Deutschen nach Interventionsmaßnahme in dieser Sprache) erfüllt sind.

\subsection{Zusammenhänge bezüglich der Textqualität im Deutschen und Türkischen}

Die Berechnungen der Assoziationen (Spearman-Korrelationen) zwischen der Textqualität im Deutschen und im Türkischen ergaben stets signifikante Zusammenhän-

Tab. 3 Nichtstandardisierte Effekte und 90\%-Konfidenzintervalle der direkten und indirekten Effekte in Mediationsmodellen der Interventionsgruppen

\begin{tabular}{|c|c|c|c|c|}
\hline \multirow[b]{2}{*}{ Gruppe $^{\mathrm{a}}$} & \multicolumn{4}{|c|}{ Nichtstandardardisierte Effekte [90\%-Konfidenzintervalle] } \\
\hline & $\begin{array}{l}\text { Direkter Effekt (c'- } \\
\text { Pfad) }\end{array}$ & a-Pfad & b-Pfad & $\begin{array}{l}\text { Indirekter Effekt } \\
(\mathrm{a} * \mathrm{~b})\end{array}$ \\
\hline $\begin{array}{l}\text { Ausdruck } \\
(n=19)\end{array}$ & $\begin{array}{l}0,069 \\
{[-0,072 ; 0,021]}\end{array}$ & $\begin{array}{l}-0,014 \\
{[-0,123 ; 0,094]}\end{array}$ & $\begin{array}{l}0,744 \\
{[0,381 ; 1,108]}\end{array}$ & $\begin{array}{l}-0,011 \\
{[-0,135 ; 0,060]}\end{array}$ \\
\hline $\begin{array}{l}\text { Schema } \\
(n=26)\end{array}$ & $\begin{array}{l}0,106 \\
{[-0,031 ; 0,242]}\end{array}$ & $\begin{array}{l}0,190 \\
{[0,091 ; 0,289]}\end{array}$ & $\begin{array}{l}0,545 \\
{[0,228 ; 0,863]}\end{array}$ & $\begin{array}{l}\mathbf{0 , 1 0 3} \\
{[0,043 ; 0,181]}\end{array}$ \\
\hline $\begin{array}{l}\text { Prozedur } \\
(n=24)\end{array}$ & $\begin{array}{l}0,127 \\
{[-0,016 ; 0,270]}\end{array}$ & $\begin{array}{l}0,168 \\
{[0,044 ; 0,392]}\end{array}$ & $\begin{array}{l}0,488 \\
{[0,202 ; 0,774]}\end{array}$ & $\begin{array}{l}\mathbf{0 , 0 8 2} \\
{[0,021 ; 0,179]}\end{array}$ \\
\hline
\end{tabular}

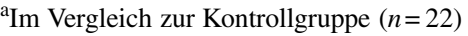


ge, meist von mittlerer Größe (mit ** für $p<0,01$ und * für $p<0,05$ ): MZP 1: $r_{s}=0,22^{*}, \operatorname{MZP} 2: r_{s}=0,53^{* *}, \operatorname{MZP} 3: r_{s}=0,40^{* *}$, MZP 4: $r_{s}=0,27 *$, MZP 5: $r_{s}=0,45^{* *}, \operatorname{MZP} 6: r_{s}=0,55^{* *}, \operatorname{MZP} 7: r_{s}=0,34^{* *}$.

Die Hypothese 2 bestätigt sich: Es gibt statistisch signifikante interlinguale, meist mittlere Zusammenhänge bezüglich der Textqualität zwischen dem Deutschen und Türkischen.

\subsection{Zusammenhänge bezüglich der Revisionshandlungen im Deutschen und Türkischen}

Es gibt statistisch signifikante Zusammenhänge zwischen den Revisionshandlungen im Deutschen und Türkischen. Je nach betroffener Textprozedur war die Auftretenswahrscheinlichkeit einer Revisionshandlung unterschiedlich erhöht. So erhöhte das Streichen im Deutschen die Auftretenswahrscheinlichkeit im Türkischen um das 32- bis 118-fache, das Übernehmen um das 60- bis 150-fache, das Verändern um das 19- bis 50-fache, das Ersetzen um das 10- bis 18-fache und das Erweitern um das 23- bis 48-fache. Da keine relevanten Interaktionseffekte in Bezug auf die Textprozeduren vorlagen, war dieser Befund nicht von der jeweils zu überarbeitenden Textprozedur beeinflusst. Sämtliche statistischen Kennwerte inkl. der Omnibus-Tests sind in X nachzulesen. Tab. 4 zeigt die Odds-Ratios und 95\%-Konfidenzintervalle der logistischen Regressionen nur für die Zusammenhänge zwischen den Revisionshandlungen in den deutschen Texten (unabhängige Variable) und den türkischen Texten (abhängige Variable), aufgeteilt nach Textprozedur:

Tab. 4 Zusammenhänge zwischen den Revisionshandlungen in den deutschen und türkischen Texten nach Textprozedur (Prädiktorvariable: Handlung im Deutschen)

\begin{tabular}{|c|c|c|c|c|c|}
\hline \multirow[b]{2}{*}{ Schema } & \multicolumn{5}{|c|}{ Revisionshandlung im Türkischen [95\%-Konfidenzintervalle] } \\
\hline & Ersetzen & Erweitern & Streichen & Übernehmen & Verändern \\
\hline Agentivieren & $\begin{array}{l}12,411 * * * \\
{[3,215 ;} \\
47,916]\end{array}$ & $\begin{array}{l}22,500 * * * \\
{[8,966 ;} \\
56,463]\end{array}$ & $\begin{array}{l}32,139 * * * \\
{[13,462 ;} \\
76,729]\end{array}$ & $\begin{array}{l}70,500 * * * \\
{[15,098 ;} \\
329,196]\end{array}$ & $\begin{array}{l}28,987 * * * \\
{[12,271 ;} \\
68,474]\end{array}$ \\
\hline Deuten & $\begin{array}{l}10,214 * * \\
{[2,104} \\
49,589]\end{array}$ & $\begin{array}{l}28,205 * * * \\
{[11,978 ;} \\
66,416]\end{array}$ & $\begin{array}{l}36,711 * * * \\
{[13,637} \\
98,829]\end{array}$ & $\begin{array}{l}63,111 * * * \\
{[13,286 ;} \\
299,792]\end{array}$ & $\begin{array}{l}18,958 * * * \\
{[8,219 ;} \\
43,728]\end{array}$ \\
\hline Kategorisieren & $\begin{array}{l}10,000 * * * \\
{[2,417 ;} \\
41,377]\end{array}$ & $\begin{array}{l}47,515 * * * \\
{[18,494 ;} \\
122,077]\end{array}$ & $\begin{array}{l}36,000 * * * \\
{[14,850 ;} \\
87,272]\end{array}$ & $\begin{array}{l}150,000 * * * \\
{[13,529 ;} \\
1663,068]\end{array}$ & $\begin{array}{l}39,083 * * * \\
{[15,833 ;} \\
96,476]\end{array}$ \\
\hline Kontextuieren & $\begin{array}{l}10,972 * * * \\
{[2,979 ;} \\
40,409]\end{array}$ & $\begin{array}{l}42,848 * * * \\
{[17,757 ;} \\
103,396]\end{array}$ & $\begin{array}{l}63,786 * * * \\
{[23,651 ;} \\
172,029]\end{array}$ & $\begin{array}{l}69,000 * * * \\
{[15,244 ;} \\
312,329]\end{array}$ & $\begin{array}{l}42,000 * * * \\
{[17,776 ;} \\
99,237]\end{array}$ \\
\hline Vergleichen & $\begin{array}{l}13,524 * * * \\
{[3,093 ;} \\
59,129]\end{array}$ & $\begin{array}{l}42,255^{* * * *} \\
{[16,822 ;} \\
106,135]\end{array}$ & $\begin{array}{l}117,700 * * * \\
{[35,045 ;} \\
395,295]\end{array}$ & $\begin{array}{l}87,600 * * * \\
{[14,027 ;} \\
547,085]\end{array}$ & $\begin{array}{l}46,582 * * * \\
{[17,673 ;} \\
122,778]\end{array}$ \\
\hline Verorten & $\begin{array}{l}17,875 * * * \\
{[3,763 ;} \\
84,905]\end{array}$ & $\begin{array}{l}43,636 * * * \\
{[15,583 ;} \\
122,196]\end{array}$ & $\begin{array}{l}45,324 * * * \\
{[17,930 ;} \\
114,569]\end{array}$ & $\begin{array}{l}60,000 * * * \\
{[13,240 ;} \\
271,913]\end{array}$ & $\begin{array}{l}50,104 * * * \\
{[19,288 ;} \\
130,156]\end{array}$ \\
\hline
\end{tabular}

Mit *** für $p<0,001$ und ** für $p<0,01$ 
Somit bestätigt sich auch die Hypothese 3: Die Ergebnisse der logistischen Regressionen zeigen, dass sehr starke Zusammenhänge zwischen den Revisionshandlungen in den deutschen und türkischen Texten bestanden; wurde eine Revisionshandlung an einer Textprozedur im deutschen Text durchgeführt, war die Wahrscheinlichkeit, dass die gleiche Revisionshandlung an dieser Textprozedur im türkischen Text durchgeführt wurde, um das 10- bis 150-fach erhöht.

\section{Diskussion und Ausblick}

In diesem Aufsatz wird angenommen, dass plurilinguale Kompetenzen wesentlich durch interlinguale Zusammenhänge gekennzeichnet sind. Der Erwerb dieser Kompetenzen ist demnach als lebenslanger, dynamischer Prozess zu verstehen, bei der Kompetenzen, die in einer Einzelsprache erworben werden, den Erwerb von Kompetenzen in anderen Einzelsprachen moderieren können. Es wird außerdem angenommen, dass dieses interlinguale Potenzial für einen sprachfächerverbindenden Unterricht genutzt werden kann, indem bei derjenigen Einzelsprache angesetzt wird, in der die betreffenden Kompetenzen der Schüler*innen am weitesten entwickelt sind, und Teilkompetenzen zum Gegenstand gemacht werden, die sprachenübergreifend relevant sind.

Diese Thesen werden durch die im vorliegenden Beitrag vorgestellten Ergebnisse für den Schreibunterricht bestätigt. Schreibfördermaßnahmen, die im Deutschunterricht materialgestützt zu sprachenübergreifenden Textprozedurenschemata des Beschreibens durchgeführt wurden (z.B. Vergleichen), führten über die Änderungen in den deutschen Texten zu signifikant besseren Beschreibungen im Türkischunterricht. Von materialgestützten Fördermaßnahmen, die sich auf einzelsprachliche Textprozedurenausdrücke beschränkten (z. B. „A sieht aus wie B“), profitierten die Schüler*innen hingegen nicht. Darüber hinaus zeigten sich signifikante interlinguale Zusammenhänge zwischen dem deutschen und dem türkischen Schreiben. Dies betraf zum einen das Schreibprodukt: Schüler*innen, die im Deutschen gute Texte schrieben, schrieben auch im Türkischen gute Texte. Zum anderen betraf es den Schreibprozess: Schüler*innen, die im Deutschen bestimmte Revisionshandlungen bevorzugten, bevorzugten diese auch im Türkischen. Hervorzuheben ist, dass die Effekte und die Zusammenhänge für eine synthetische und eine agglutinierende Sprache nachgewiesen werden konnten, also über erhebliche sprachstrukturelle Unterschiede hinweg.

Dabei muss bedacht werden, dass die Ergebnisse aus einem Schreibunterricht hervorgegangen sind, der unter quasi-experimentellen Bedingungen stattfand. $\mathrm{Ob}$ reguläre Unterrichtsbedingungen zu in Form und Ausprägung ähnlichen Ergebnissen führen, müssen weitere Studien klären. Man darf diesbezüglich aber optimistisch sein, weil die Interventionsstudie im Regelunterricht stattfand und außerdem rein materialbasiert war: Den Schüler*innen standen lediglich Arbeitsblätter und ein Stift zur Verfügung, und ihr Schreibprozess wurde weder durch eine Lehrkraft noch durch Mitschüler*innen unterstützt. Mit einem gezielten Schreibtraining und geschulten Lehrer*innen sollte im Vergleich zur Intervention eher mehr als weniger bewirkt werden können. 
Für ein solches Training haben wir eine umfangreiche Broschüre entwickelt (vgl. Reichert et al. 2020). Sie schließt an das im Projekt eingesetzte Material zum Beschreiben von Superheld*innen und -schurk*innen an, baut es zu einer sprachfächerverbindenden Unterrichtsreihe für den Deutsch-, Türkisch- und Englischunterricht in der 5., 6. und 7. Klasse aus und ist hier zugänglich: https://www. mehrsprachigkeit.uni-hamburg.de/oeffentlichkeit/materialien/textueberarbeitung.

html. Die Broschüre wird durch die in den Abschn. 3.3 und 3.4 thematisierten Lernvideos zum Überarbeiten ergänzt, die hier abgerufen werden können: https:// www.youtube.com/watch?v=QZ7fNf0Ih8c (Deutsch), https://www.youtube.com/ watch? $\mathrm{v}=\mathrm{nBoNavkR} 4 \mathrm{tA}$ (Türkisch).

Um das enorme Lernpotential interlingualer Kompetenzen für die Schule nutzbar zu machen, bedarf es nach unserem Dafürhalten, über die dargestellten Ergebnisse hinausgehend, einer Reihe von Einsichten, z.B. dass

- bi- bzw. plurilinguale Schüler*innen, die in Deutschland eingeschult worden sind, in der deutschen Sprache höhere bildungssprachliche Sprachkompetenzen als in ihren nichtdeutschen Sprachen besitzen,

- der Erwerb sprachlicher Kompetenzen Teil eines plurilingualen Spracherwerbs ist, dessen erfolgreiche Bewältigung erhebliche Vorteile für die Bildungs- und berufliche Laufbahn der Schüler*innen in einer multikulturellen Gesellschaft birgt und außerdem positiv auf den Erwerb des Deutschen zurückwirkt,

- der Schulung verbreiteter nichtdeutscher Familiensprachen (Türkisch, Polnisch, Russisch etc.) mehr Aufmerksamkeit geschenkt werden sollte,

- neben mündlichen auch Lese- und Schreibkompetenzen vermittelt werden sollten und

- Deutsch-, Fremd- und Herkunftssprachenlehrer*innen eng zusammenarbeiten und sprachenübergreifend effektive Lernarrangements entwickeln sollten, die sie dann gemeinsam und regelmäßig umsetzen.

Funding Open Access funding enabled and organized by Projekt DEAL.

Open Access Dieser Artikel wird unter der Creative Commons Namensnennung 4.0 International Lizenz veröffentlicht, welche die Nutzung, Vervielfältigung, Bearbeitung, Verbreitung und Wiedergabe in jeglichem Medium und Format erlaubt, sofern Sie den/die ursprünglichen Autor(en) und die Quelle ordnungsgemäß nennen, einen Link zur Creative Commons Lizenz beifügen und angeben, ob Änderungen vorgenommen wurden.

Die in diesem Artikel enthaltenen Bilder und sonstiges Drittmaterial unterliegen ebenfalls der genannten Creative Commons Lizenz, sofern sich aus der Abbildungslegende nichts anderes ergibt. Sofern das betreffende Material nicht unter der genannten Creative Commons Lizenz steht und die betreffende Handlung nicht nach gesetzlichen Vorschriften erlaubt ist, ist für die oben aufgeführten Weiterverwendungen des Materials die Einwilligung des jeweiligen Rechteinhabers einzuholen.

Weitere Details zur Lizenz entnehmen Sie bitte der Lizenzinformation auf http://creativecommons.org/ licenses/by/4.0/deed.de. 


\section{Literatur}

Anskeit, N. (2019). Schreibarrangements in der Primarstufe. Eine empirische Untersuchung zum Einfluss der Schreibaufgabe und des Schreibmediums auf argumentative und deskriptive Texte und Schreibprozesse in der 4. Klasse. Münster: Waxmann.

Bachmann, T., \& Becker-Mrotzek, M. (2010). Schreibaufgaben situieren und profilieren. In T. Pohl \& T. Steinhoff (Hrsg.), Textformen als Lernformen (S. 5-26). Duisburg: Gilles \& Francke.

Baker, C. (2011). Foundations of bilingual education and bilingualism (5. Aufl.). Bristol: Multilingual Matters.

Berthele, R., \& Lambelet, A. (2018). Investigating interdependence and literacy development in heritage language speakers: theoretical and methodological considerations. In R. Berthele \& A. Lambelet (Hrsg.), Heritage and school language literacy development in migrant children: interdependence or independence? (S. 1-25). Clevedon: Multilingual Matters.

Brehmer, B., \& Mehlhorn, G. (Hrsg.) (2015). Themenheft „Herkunftssprachen“. Zeitschrift für Fremdsprachenforschung, 26(1), 3-6.

Cummins, J. (2000). Language, power, and pedagogy. Bilingual children in the crossfire. Clevedon: Multilingual Matters.

Cummins, J. (2010). Language support for pupils from families with migration backgrounds: challenging monolingual instructional assumptions. In C. Benholz, G. Kniffka \& E. Winters-Ohle (Hrsg.), Fachliche und sprachliche Förderung von Schülern mit Migrationsgeschichte: Beiträge des MercatorSymposions im Rahmen des 15. AILA-Weltkongresses „Mehrsprachigkeit: Herausforderungen und Chancen" (S. 13-23). Münster: Waxmann.

Feilke, H. (2014). Argumente für eine Didaktik der Textprozeduren. In T. Bachmann \& H. Feilke (Hrsg.), Werkzeuge des Schreibens. Beiträge zu einer Didaktik der Textprozeduren (S. 11-34). Stuttgart: Klett.

Fix, M. (2000). Textrevisionen in der Schule. Prozessorientierte Schreibdidaktik zwischen Instruktion und Selbsteuerung. Empirische Untersuchungen in achten Klassen. Baltmannsweiler: Schneider Hohengehren.

Hayes, A. F. (2018). Introduction to mediation, moderation, and conditional process analysis. A regressionbased approach (2. Aufl.). Guilford: New York, London.

Juhasz, J. (1970). Probleme der Interferenz. München: Hueber.

Marx, N. (2014). Häppchen oder Hauptgericht? Zeichen der Stagnation in der deutschen Mehrsprachigkeitsdidaktik. Zeitschrift für interkulturellen Fremdsprachenunterricht, 1, 8-24.

Marx, N. (2017). Schreibende mit nichtdeutscher Familiensprache. In M. Becker-Mrotzek, J. Grabowski \& T. Steinhoff (Hrsg.), Forschungshandbuch empirische Schreibdidaktik (S. 139-152). Münster: Waxmann.

Marx, N. (2020). Transfer oder Transversalität? - Zur Erforschung der Mehrschriftlichkeit. Bulletin VALSASLA, Printemps, 15-33. http://doc.rero.ch/record/11876/files/bulletin_vals_asla_2020_special.pdf

Marx, N., \& Steinhoff, T. (2017a). Unterrichtsbezogene Interventionen. In M. Becker-Mrotzek, J. Grabowski \& T. Steinhoff (Hrsg.), Forschungshandbuch empirische Schreibdidaktik (S. 253-266). Münster: Waxmann.

Marx, N., \& Steinhoff, T. (2017b). Schlussbericht zu dem vom BMBF geförderten Forschungsprojekt „Schreibförderung in der multilingualen Orientierungsstufe. Zur Wirksamkeit des wiederholten Einsatzes unterschiedlich profilierter Revisionsarrangements auf die Textproduktion von Schülerinnen und Schülern der 6. Jahrgangsstufe in Oberschulen, Gesamtschulen und Gymnasien in den Erstsprachen Deutsch und Türkisch und in der Zweitsprache Deutsch. https://www.tib.eu/de/suchen/id/ TIBKAT\%3A886945909/. Zugegriffen: 03. Mai 2021.

Marx, N., \& Steinhoff, T. (2020). Textrevisionen in der multilingualen Orientierungsstufe. https://www.tib. eu/en/suchen/id/TIBKAT:1734614803/.. Zugegriffen: 3. Mai 2021.

Odlin, T. (1989). Language transfer. Cross-linguistic influence in language learning. Cambridge: CUP.

Rat der Europäischen Union (2015). Schlussfolgerungen des Rates vom 20. Mai 2014 zur Mehrsprachigkeit und zur Entwicklung von Sprachenkompetenz. https:/op.europa.eu/de/publication-detail/-/ publication/18fb5e89-f38a-11e3-831f-01aa75ed71a1. Zugegriffen: 3. Mai 2021.

Reichert, M.-C., \& Marx, N. (2020). Mehrsprachige Schreibende - mehrsprachiges Schreiben? Fremdsprachen Lehren und Lernen (flul), 49(1), 36-50.

Reichert, M.-C., Steinhoff, T., \& Marx, N. (2020). Texte überarbeiten! Lehrerkommentar und Materialien zur Schulung der Überarbeitungsfähigkeiten. https://www.mehrsprachigkeit.uni-hamburg.de/ oeffentlichkeit/materialien/textueberarbeitung.html. Zugegriffen: 3. Mai 2021. 
Rüßmann, L. (2018). Schreibförderung durch Sprachförderung. Eine Interventionsstudie zur Wirksamkeit sprachlich profilierter Schreibarrangements in der mehrsprachigen Sekundarstufe I. Münster: Waxmann.

Singleton, D., \& Aronin, L. (Hrsg.). (2019). Twelve lectures on multilingualism. Bristol: Multilingual Matters.

Souvignier, E., Trenk-Hinterberger, I., Adam-Schwebe, S., \& Gold, A. (2008). FLVT 5-6. Frankfurter Leseverständnistest für 5. und 6. Klassen. Göttingen: Hogrefe.

Steinhoff, T. (2018). Schreibarrangements. Impulse für einen lernförderlichen Schreibunterricht. Der Deutschunterricht, 3, 2-10.

Stevenson, M., Schoonen, R., \& de Glopper, K. (2006). Revising in two languages: a multi-dimensional comparison of online writing revisions in L1 and FL. Journal of Second Language Writing, 15, 201-233.

telc (o.J.). https://www.telc.net/shop/tuerkisch.html. Zugegriffen: 3. Mai 2020.

Wenk, A.K. (2018). Bilinguale Schreibförderung. Eine Interventionsstudie zur Wirksamkeit sprachlich unterschiedlich profilierter Schreibarrangements im Deutschunterricht auf die Textqualität in der Familiensprache Türkisch bei bilingualen Schülerinnen und Schülern der Sekundarstufe I. Bremen: Universität Bremen.

Wenk, A. K., Marx, N., Rüßmann, L., \& Steinhoff, T. (2016). Förderung bilingualer Schreibfähigkeiten am Beispiel Deutsch-Türkisch. Zeitschrift für Fremdsprachenforschung, 27(2), 151-179. 